\section{Myeloid Growth Factor Guidelines: Moving Toward a Societal Perspective}

The Myeloid Growth Factors guidelines in this month's issue are in some ways a paradigm for successful guidelines—guidelines that support clinical decision-making. The algorithm comes down to the level of actual administration, with specific thresholds for beginning, dosages, and stopping. This granularity is possible, of course, because of the extraordinary number of trials meticulously designed to answer specific drug-related questions. For example, Frankfurt and Tallman's article on using these agents in leukemia describes trials that have investigated not only the efficacy of these stimulating factors but also their potential deleterious effects. I'm reassured to learn from Lyman and Kleiner of the consistency found across 3 well-known guidelines for these agents.

A major recommendation in all 3 guidelines is that growth factors be initiated for primary prophylaxis if the expected rate of febrile neutropenia is $20 \%$. This new threshold varies considerably from the initial American Society of Clinical Oncology (ASCO) guidelines in 1994, which used a 40\% threshold. The new mark is based on results from several randomized clinical trials and meta-analyses that confirm the efficacy of these agents in significantly diminishing the incidence of febrile neutropenia at this level.

In what will likely become a major issue for guidelines developers in the future, economic considerations emerged in these discussions. Although NCCN and ASCO state that decisions were based on proven clinical benefit, both groups acknowledge the potentially large economic impact that using these agents may have- both their capacity to save in-hospital expenses and the financial burden they could impose if large numbers of patients are treated with them. This is especially concerning when only a minority of patients may actually benefit.

One of the major goals of clinical practice guidelines is improving the quality of care. In formulating goals of quality care, the Institute of Medicine proposed that quality be based not only on effectiveness but also on the efficiency of care. Many professional groups, such as the National Quality Forum and the Ambulatory Quality Alliance, now focus considerable resources on developing a framework for how these economic issues will be defined and integrated into value-based systems of health care. The concept is not new, and our hospitals have been operating under a Diagnostic Related Groups (DRG) system that is designed to promote efficiency.

Efficiency relates to the provision of optimal quality care with the optimal use of resources. ASCO guidelines recognize this in the statement that if alternative chemotherapy regimens are available that produce the same result without the need for growth factors, they should be used. That we now face a tremendous explosion of healthcare costs from new agents that significantly improve patient care or outcomes but carry costs that must be borne by the entire system is well-publicized. How to meld this societal perspective into guidelines will undoubtedly become a serious challenge to tomorrow's panels.

Bringing the dimension of cost into guidelines-an action not only appropriate but also necessary-will require new research and methodologies. The science of costeffectiveness, at least as it translates to the clinician, is still abstruse and fraught with multiple interpretations. We need newly trained oncology minds who understand not only the intricacies of the cell cycle but also the arcana of indirect costs.

The bottom line, however, is that in the end we will have better guidelines, ones in which clinical decision-making includes the full range of factors that affect our daily lives.

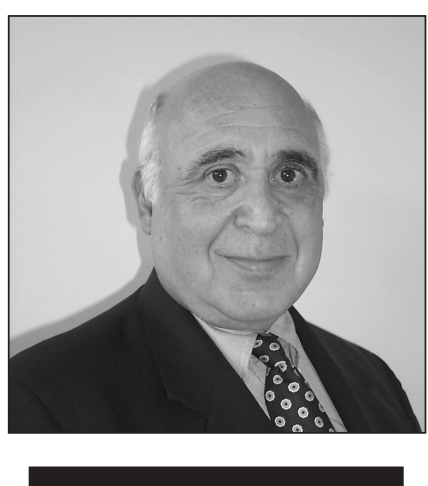

By Rodger J. Winn, MD

Rodger J. Winn is the Editorin-Chief of JNCCN. He is Clinical Consultant at the National Quality Forum, and his past positions include Associate Professor of Clinical Medicine at The University of Texas M. D. Anderson Cancer Center. Dr. Winn received his medical degree from Jefferson Medical College of Philadelphia. His postgraduate training includes an internship and residency at Jefferson Medical College, and he also completed a medical oncology fellowship at Memorial Sloan-Kettering Cancer Center in New York. He is board certified in internal medicine and holds subspecialty certification in oncology. 